\title{
Zwischenjahr mit Herz
}

\section{Adrian Ritter}

Freier Journalist

\author{
Laetitia Guarino hat die Krone als Miss Schweiz weitergereicht. Jetzt widmet sie \\ sich wieder ihrem Medizinstudium. Das Jahr als Miss Schweiz hat sie als wertvolle \\ Horizonterweiterung erlebt, gerade auch in Bezug auf das Thema Gesundheit.
}

Wie läuft eine Geburt ab? Worauf muss man bei einem Kaiserschnitt achten? Und was tun, wenn sich das Kind in Steisslage befindet? Laetitia Guarino kommt gerade aus der vierstündigen Vorlesung "Mère - Enfant», als ich sie an einem Oktobermorgen vor dem Centre hospitalier universitaire vaudois (CHUV) in Lausanne treffe. Die 23-jährige Waadtländerin befindet sich im ersten Jahr des Masterstudiums Medizin.

An jenem Oktobermorgen ist sie noch für eine letzte Woche Miss Schweiz. Es ist eine strenge Zeit. Das Medizinstudium hat sie nach einem Jahr Pause bereits im September wieder aufgenommen. Gleichzeitig nimmt sie noch zahlreiche Termine als Miss Schweiz wahr.

Das Konzept «Miss Schweiz» ist 2014 im Sinne einer «Krone mit Herz» angepasst worden. Ziel ist es insbesondere, im Amtsjahr möglichst viel Aufmerksamkeit und Unterstützung für soziale Anliegen und Organisationen zu generieren. So ist die Miss Schweiz neu Botschafterin der Stiftung «Corelina» des Berner Herzchirurgen Thierry Carrel. Die Stiftung ermöglicht herzkranken Kindern aus der Schweiz und dem Ausland medizinische Behandlung und Betreuung. Zum neuen

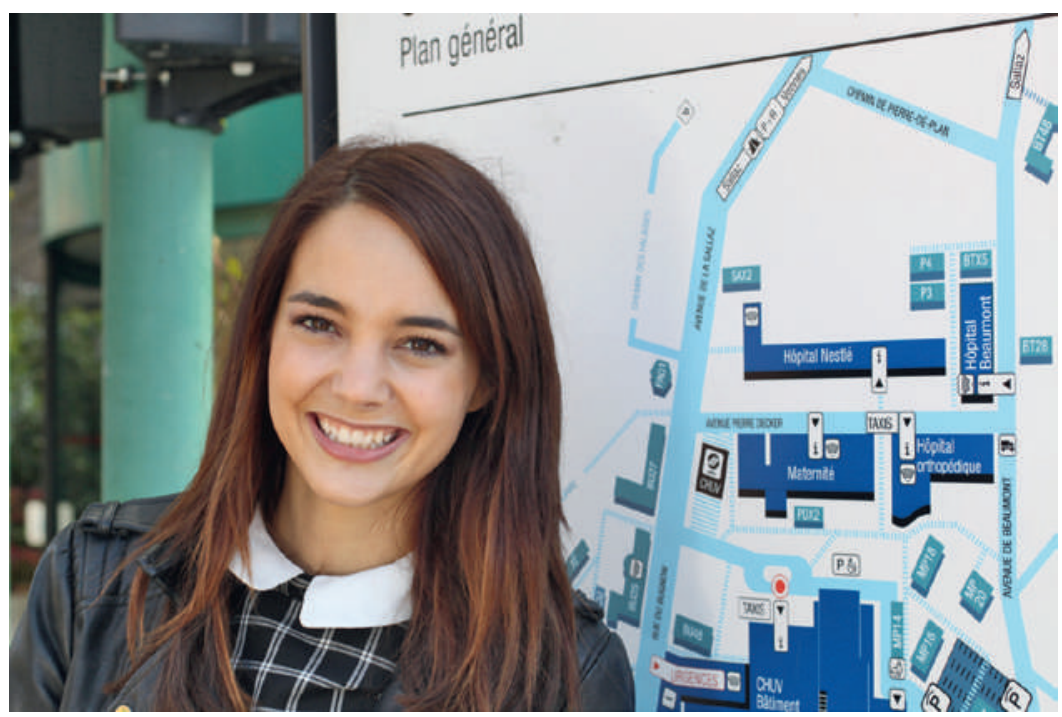

Laetitia Guarino, Miss Schweiz des Jahres 2015, vor dem Universitätsspital Lausanne.
Konzept gehört, dass die Miss Schweiz einen Fixlohn von 10000 Franken pro Monat bezieht - zusätzliche Einnahmen etwa aus Model-Aufträgen gehen an die Stiftung Corelina.

\section{Herzoperationen für die Jüngsten}

Laetitia Guarino war schon vor ihrer Wahl zur Miss Schweiz ehrenamtlich tätig. Sie engagiert sich beispielsweise in einer Organisation, die das Spenden von Blutstammzellen fördern will. «Bevor ich Miss Schweiz war, schrieb ich einmal die Medien an, ob sie darüber berichten möchten. Ich bekam keine einzige Antwort. Als ich die Medien später als Miss Schweiz kontaktierte, war das Echo deutlich grösser», erzählt sie lachend.

Während ihres Amtsjahres konnte Laetitia Guarino Erfahrungen sammeln, die ihr auch für das Medizinstudium nützlich sind. So reiste sie mit der Stiftung Corelina nach Marokko und Tunesien. Sie half in der marokkanischen Hauptstadt Rabat bei Ultraschalluntersuchungen und assistierte bei fünf Operationen an Kinderherzen - sie reichte Instrumente und saugte Blut ab. Aus Tunesien begleitete sie Kinder in die Schweiz und assistierte auch im Inselspital Bern bei Herzoperationen für die Jüngsten. Die Studienwahl von Laetitia Guarino macht es möglich ...

Es waren in Nordafrika bisweilen belastende Erfahrungen - wenn Laetitia Guarino etwa mitansehen musste, wie Eltern, die Hunderte von Kilometern ins Spital zurückgelegt hatten, wieder nach Hause geschickt wurden. Die medizinische Infrastruktur reichte nicht für die Behandlung aller Kinder, die sie benötigen. «Ich habe viel geweint in diesen zwei Wochen", sagt sie. Um das Erlebte zu verarbeiten, blieb ihr nur eins: sich auf diejenigen Kinder zu konzentrieren, denen geholfen werden konnte. Umso mehr ist sie von der Notwendigkeit karitativer Organisationen überzeugt: «Ich habe jetzt einen viel genaueren Einblick, was diese Organisationen alles tun.» Eindruck gemacht hat ihr auch die Reise mit Terre des Hommes und Corelina nach Kam- 


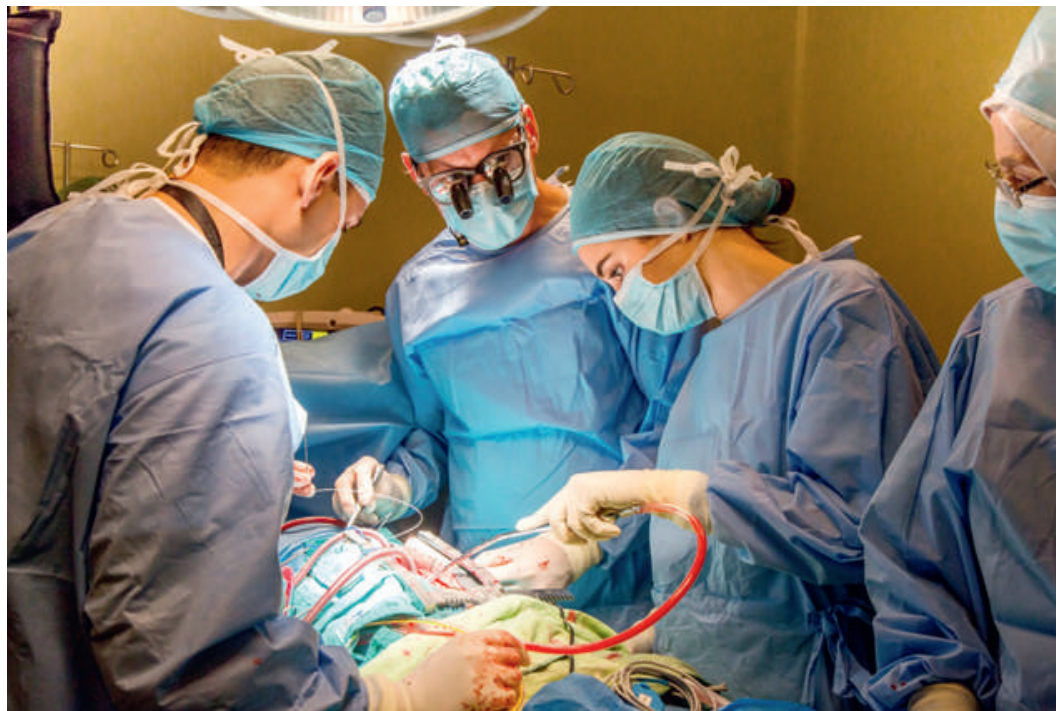

Das kann nicht jede Miss Schweiz: Als Medizinstudentin durfte Laetitia Guarino für die Stiftung Corelina auch bei Herzoperationen assistieren.

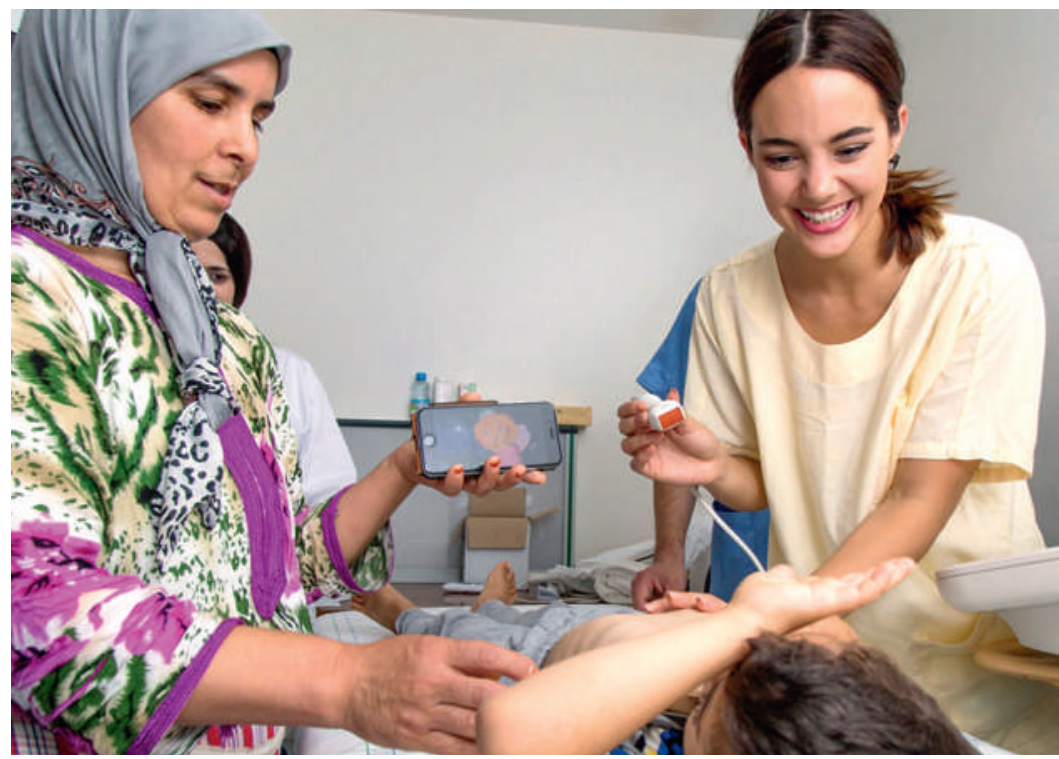

Ultraschall bei herzkranken Kindern: Während ihres Amtsjahres als Miss Schweiz sam melte Laetitia Guarino Erfahrungen, die ihr auch für das weitere Studium nützlich sind.

bodscha, wo sie mithalf, Nahrungsmittel in Slums zu verteilen. "Einmal mehr sah ich dort, wie wichtig und auch kostengünstig präventive Massnahmen zur Erhaltung der Gesundheit sind - etwa im Bereich Hygiene und Ernährung», so Guarino.

Reisen in zahlreiche Länder, Begegnungen mit Menschen aus Showbusiness, Mode, Medien und Politik: «Das Jahr als Miss Schweiz hat meinen Horizont stark erweitert», so Guarino. «Die Medizin ist manchmal eine etwas in sich abgeschottete Welt, die einen zeitlich wie mental sehr beansprucht und vereinnahmt.» Gerade das erste Jahr Bachelorstudium an der Universität Lausanne sei intensiv gewesen. Der fehlende Numerus Clausus im Medizinstudium an den Univer- sitäten der Romandie werde durch eine Vielzahl von Prüfungen ersetzt und sorge für einen Grossandrang zum Studium: «Wir waren manchmal 600 Studierende im Hörsaal», so Guarino.

Als Kind wollte Laetitia Guarino Tierärztin werden. «Ich glaube, Tiere mögen Veterinärmediziner nicht so sehr, deshalb habe ich mich dann für die Humanmedizin entschieden", erzählt sie schmunzelnd. Ein erstes Mal vertiefte sie sich im Gymnasium in ein medizinisches Thema. Sie widmete eine Semesterarbeit der Krankheit, unter der ihr Götti leidet, der Amyotrophen Lateralsklerose (ALS). Noch viel mehr praktische Erfahrung mit Menschen mit gesundheitlichen Problemen konnte sie während des Bachelorstudiums sammeln. Guarino finanzierte sich das Studium, indem sie daneben als Nachtwache in einer Institution für Menschen mit einer körperlichen Behinderung arbeitete.

\section{Verspätete Operationen}

Als sie sich für die Teilnahme am Miss-Schweiz-Wettbewerb entschied, war das als Spass gedacht, der Sieg dann eine umso grössere Überraschung. Das Jahr als Miss Schweiz wird sich weiterhin auf das wieder aufgenommene Medizinstudium auswirken. So hat sich aus dem Einblick in das Gesundheitswesen von Entwicklungsländern das Thema für ihre Masterarbeit ergeben. «Ich will am Beispiel Marokkos der Frage nachgehen, welche Auswirkungen eine späte Operation der angeborenen Herzfehlbildung Fallot-Tetralogie hat.» Während etwa in der Schweiz eine Operation in den ersten Lebensmonaten der Kinder angestrebt wird, findet eine solche in Marokko oft erst mit sechs oder sieben Jahren statt. Die Infrastruktur fehlt, um bereits Säuglinge operieren zu können. Ihre Masterarbeit will Laetitia Guarino noch in diesem Jahr beginnen und im Sommer 2016 dazu nochmals nach Marokko reisen. Wie es nach dem Studium weitergeht, ist noch offen. Bisher war für Guarino klar, dass sie Kinderärztin werden möchte. Die Erfahrungen mit der Stiftung Corelina haben ihr Interesse für die Chirurgie geweckt. Nicht zuletzt gefällt ihr auch die medizinische Forschung. Den direkten Kontakt mit den Patientinnen und Patienten allerdings würde sie vermissen: «Es fasziniert mich, ihre Geschichten zu hören und herauszufinden, was ihnen fehlt.» Zeit genug bleibt, sich in den kommenden drei Jahren zu entscheiden. Am 7. November 2015 hat Laetitia Guarino die Miss-SchweizKrone an ihre Nachfolgerin übergeben und konzentriert sich wieder ganz auf das Studium.

Bildnachweis

Porträt erste Seite: Adrian Ritter; andere: zVg (Miss Schweiz) 